\title{
TRANES analysis of the fluorescence of nile red in organized molecular assemblies confirms emission from two species
}

\author{
A S R KOTI and N PERIASAMY* \\ Department of Chemical Sciences, Tata Institute of Fundamental Research, \\ Homi Bhabha Road, Colaba, Mumbai 400 005, India \\ e-mail: peri@tifr.res.in; peri@mailhost.tifr.res.in
}

MS received 26 February 2001

\begin{abstract}
Time-resolved area normalized emission spectroscopy (TRANES) is a new method for the analysis of fluorescence of dyes in complex chemical and biological systems (A S R Koti, M M G Krishna and N Periasamy, 2001, J. Phys. Chem. 105, 1767). The model-free method extends the power of time-resolved emission spectroscopy (TRES) analysis and removes the ambiguity in the interpretation when the emission spectrum is time-dependent. Observation of an isoemissive point in TRANES analysis of fluorescence is an unambiguous indication for the presence of two emissive species in the sample. The isoemissive point occurs at a wavelength where the ratio of the radiative rates of the two species is equal to the ratio of their total radiative rates. The polarity-sensitive nile red dye shows timedependent emission spectra in the organized bilayer assemblies of TX micelle and bilayer egg-phosphotidylcholine (egg-PC) membrane. Time-dependent spectra in complex systems support many important models (solvation model and heterogeneity in the ground and/or excited state). TRANES analysis shows that the fluorescence emission of nile red in TX micelle and egg-PC membrane is due to two emissive species solubilized in different sites.
\end{abstract}

Keywords. TRANES; TRES; fluorescence; nile red; solvent relaxation; organized molecular assemblies; solvation dynamics.

\section{Introduction}

Fluorescence-based methods are very useful for the study of structure and dynamics in complex chemical and biological systems using intrinsic or extrinsic fluorescent probes ${ }^{1}$. A polarity- and viscosity-sensitive dye such as nile red is used to probe the environmental properties around the site of solubilization in organized molecular assemblies of micelles and bilayer membranes, and biological complex systems. Knowledge of the number of emissive species is important for the interpretation of the fluorescence properties associated with each species and their correlation with the structure and dynamics of each species. In most cases, fluorescence decay is multiexponential and the emission spectrum is time-dependent. It is possible to identify the number of emissive species without assuming a priori models only in some cases ${ }^{2}$.

Construction of time-resolved emission spectra (TRES) is a useful method ${ }^{1}$. If the emission spectrum is the same at all times, then a single emissive species can be inferred.

*For correspondence 
It may be noted that fluorescence decay may be multiexponential even when a single emissive species is present. For example, when bimolecular fluorescence quenching is present, emission is due to a single species but the emission decay is not a single exponential $^{3}$. When TRES shows that the emission spectrum is time-dependent, multiple emissive species are indicated. We have shown recently that construction of timeresolved area normalized emission spectra (TRANES) is useful in identifying two emissive species in the sample, irrespective of the origin of the species or mechanism of their excited state kinetics ${ }^{2}$. TRANES analysis is therefore useful for understanding the complexity in organized molecular assemblies using fluorescent probes.

There are many studies of fluorescent probes in organized molecular assemblies and biological membranes ${ }^{4-6}$. The multiexponential decay and/or time-dependent emission spectra are interpreted either as emission from two species ${ }^{4}$ or emission from multiple species (for example, solvation model) ${ }^{5,6}$. In this paper, we show that the application of TRANES method can resolve the above two models unambiguously. We apply the method for the analysis of the fluorescence of nile red in organized molecular assemblies of micelles and bilayer membranes and conclude that the emission is due to two species.

\section{Experimental}

Nile red (phenoxazone-9), TX-100 and egg-phosphotidylcholine (egg-PC) were purchased from Sigma Chemicals (USA) and the dye was tested to be pure by TLC. 2Octanol (Aldrich Chemicals, USA), ethanol and methanol (Spectroscopy grade, SD Fine Chemicals, Mumbai) were used as solvents in this study. Steady state fluorescence spectra were recorded using a spectrofluorimeter (SPEX model 1681T) and corrected for the spectral sensitivity of the photomultiplier (Hamamatsu R928A). Time-resolved fluorescence decays were obtained by time correlated single-photon counting (TCSPC) $\operatorname{method}^{7}$. The sample was excited by 'vertically' polarized picosecond laser pulses $(800 \mathrm{kHz}$ or $4 \mathrm{MHz})$. Fluorescence emission at magic angle $\left(54.7^{\circ}\right)$ was dispersed in a monochromator $(f / 4$, slit width $2.5 \mathrm{~nm})$ and counted $\left(3-4 \times 10^{3} \mathrm{~s}^{-1}\right)$ by a MCP PMT (R2809), and processed through CFD, TAC and MCA. The instrument response function is $\sim 50 \mathrm{ps}$.

The samples in organized molecular assemblies were prepared as follows. The $2 \%$ TX micelle solution was prepared by stirring the surfactant in warm deionized water for about $1 \mathrm{~h}$. The sonicated egg-PC liposomes were prepared in $p \mathrm{H} 7.4$ buffer $(10 \mathrm{mM}$ $\left.\mathrm{CH}_{3} \mathrm{COONa}, 10 \mathrm{mM} \mathrm{NaH}{ }_{2} \mathrm{PO}_{4}\right)$. The lipid concentration used in these experiments was $\sim 0.2 \mathrm{mg} / \mathrm{mL}(\sim 0.28 \mathrm{mM})$. The nile red dye (stock solution in methanol, $1 \mathrm{mM}$ ) was added to these organized molecular assemblies and stirred. Thus, all samples contain methanol $0.2 \%$ by volume. The samples were kept overnight for equilibration. The dye to surfactant ratio was approximately 1:25000. The dye to lipid ratio was approximately $1: 250$. All the measurements were carried out in air-saturated solutions at temperature $25^{\circ} \mathrm{C}$, which is maintained constant throughout the experiment.

\section{Results and discussion}

TRES and TRANES spectra for nile red in all the samples used in this study were obtained using the corrected steady state fluorescence spectrum and the fluorescence decays at all emission wavelengths (at $5 \mathrm{~nm}$ intervals) by the four-step procedure as described earlier ${ }^{2}$, without assuming any model for the fluorescence decay. 
Figure 1 shows the TRES and TRANES spectra for nile red in methanol. TRES spectra of nile red in methanol (figure 1a) show that the spectral peak does not shift with time from 0 to $10 \mathrm{~ns}$. TRANES spectra (figure 1b) show a single spectrum in the same time scale. The inset in figure 1 shows the peak region in greater detail. This confirms that there is only one emissive species in methanol. A single emissive species is also observed in the solvent ethanol (results are not shown).

Fluorescence properties of nile red in ethanol differ marginally from those of methanol. Fluorescence spectral peak positions and fluorescence lifetimes are $628 \mathrm{~nm}$

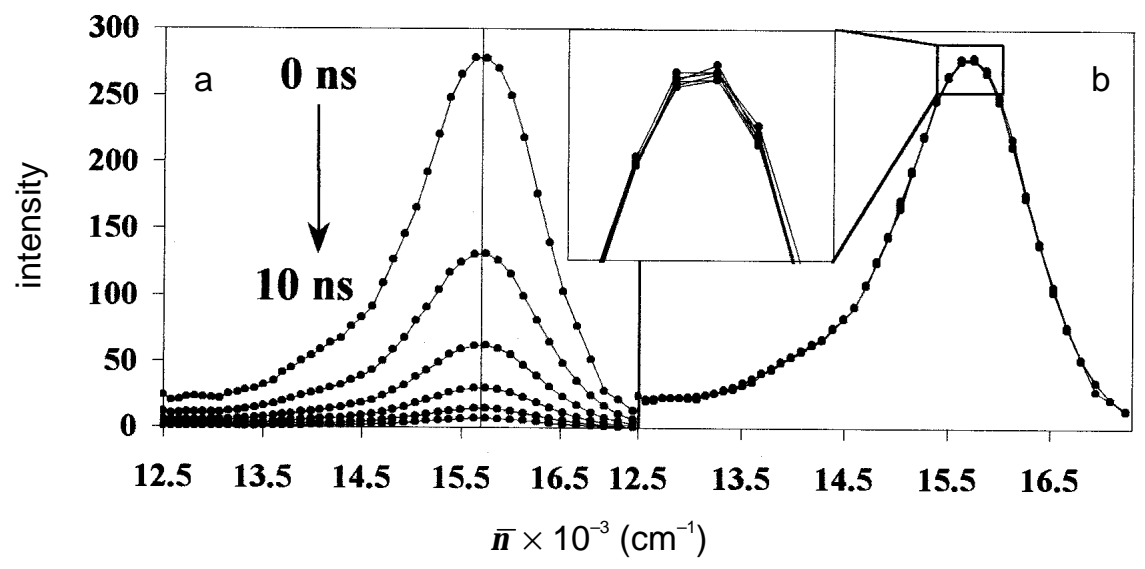

Figure 1. (a) Time-resolved emission spectra (TRES), and (b) time-resolved area normalized emission spectra (TRANES) for nile red in methanol. Inset shows the peak region in greater detail. The spectra are the same at all times. [dye] $=2 \mu \mathrm{M}$. $\lambda_{\mathrm{ex}}=571 \mathrm{~nm}$. The spectra are shown for times, $0,2,4,6,8$ and $10 \mathrm{~ns}$.

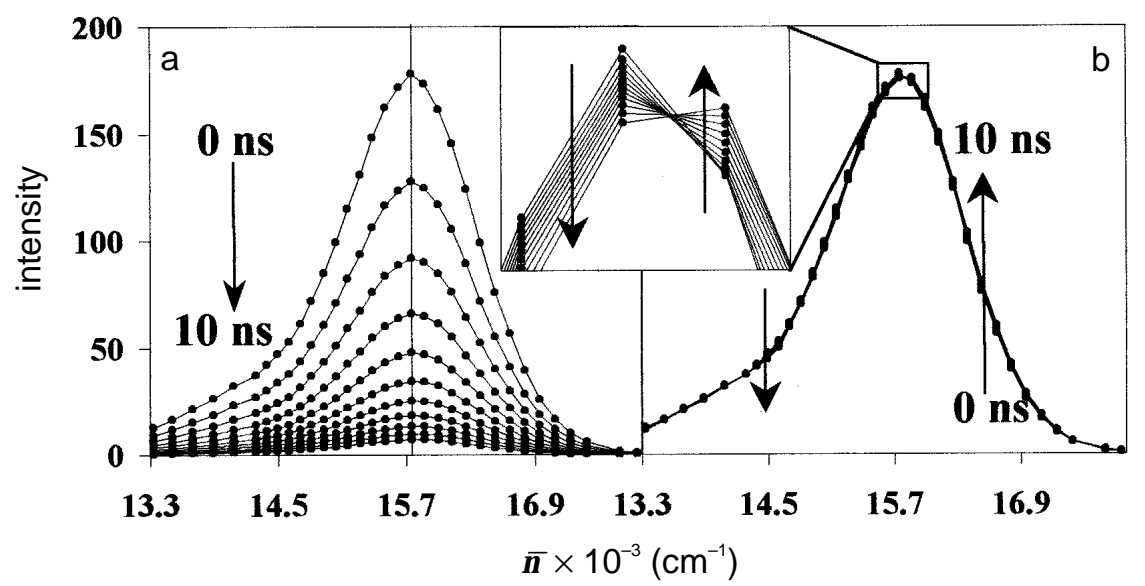

Figure 2. (a) Time-resolved emission spectra (TRES), and (b) time-resolved area normalized emission spectra (TRANES) for two nile red samples (in methanol and ethanol), simultaneously excited in a two-compartment cuvette. Inset shows the peak region in greater detail. Isoemissive point is observed at $632 \mathrm{~nm}$. [dye] $=2 \mu \mathrm{M}$. $\lambda_{\mathrm{ex}}=571 \mathrm{~nm}$. The spectra are shown for times, 0 to $10 \mathrm{~ns}$ at $1 \mathrm{~ns}$ intervals. 
and $3.58 \pm 0.03 \mathrm{~ns}$ (in ethanol) and $635 \mathrm{~nm}$ and $2.78 \pm 0.03 \mathrm{~ns}$ (in methanol). Figures $2 \mathrm{a}$ and $b$ show the TRES and TRANES spectra for a sample 'mixture', where the samples of nile red in methanol and ethanol were kept adjacent to each other in a two-compartment cuvette and both the samples were excited simultaneously. Since the spectral peaks in methanol and ethanol are close $(\sim 7 \mathrm{~nm})$, the spectral peak shift with time in the TRES and TRANES spectra (figures $2 \mathrm{a}$ and $\mathrm{b}$ ) is not apparent. The inset in figure 2 shows the peak region in greater detail. An isoemissive point is clearly indicated. The isoemissive point indicates that the fluorescence is due to two emissive species. The two emissive species in the sample 'mixture' are nile red in methanol and ethanol. In spite of the marginal difference in the fluorescence properties in the two alcohols, TRANES could identify unambiguously that nile red is in two different environments!

Figures 3 shows the TRES and TRANES spectra for nile red in 2-octanol. In this case, TRES spectra (figure 3a) show a continuous shift of spectral peak with time. TRANES spectra (figure $3 \mathrm{~b}$ and inset) also show a continuous shift of the spectral peak with time. Absence of an isoemissive point in TRANES shows that the fluorescence emission in 2octanol originates from three or more emissive species. A solvation model or any other energy relaxation model, which includes a continuous distribution of energy levels between the initial excited state and a final relaxed one, is more appropriate in this case.

The results for nile red in octanol and the sample 'mixture' establish the usefulness of TRANES in distinguishing between the two cases. The remarkable ability of TRANES to distinguish the solvent environments of nile red that are marginally different (e.g. methanol and ethanol) can be used to obtain specific information of fluorophore environments and dynamics in organized molecular assemblies.

Figures 4 shows the TRES and TRANES spectra for nile red in TX micelle. A continuous spectral peak shift $(\sim 10 \mathrm{~nm})$ in TRES spectra (figure $4 \mathrm{a})$ is observed in this case. However, an isoemissive point in TRANES is clearly observed for nile red in TX micelle (figure $4 \mathrm{~b}$ and inset) confirming that the fluorescence emission is due to two emissive species.

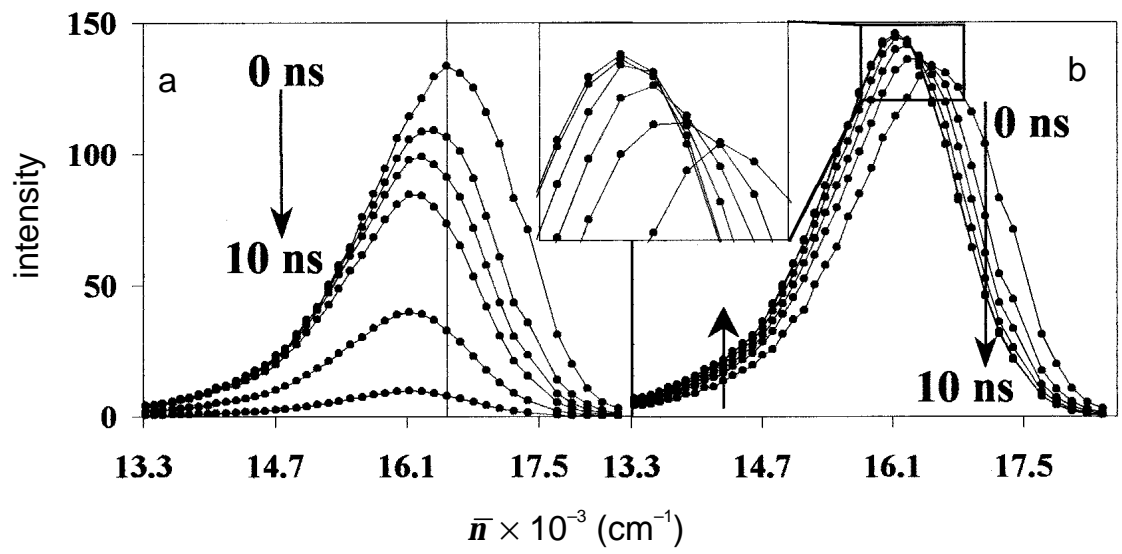

Figure 3. (a) Time-resolved emission spectra (TRES), and (b) time-resolved area normalized emission spectra (TRANES) for nile red in 2-octanol. Inset shows the peak region in greater detail. No isoemissive point is observed here. [dye] $=2 \mu \mathrm{M}$. $\lambda_{\mathrm{ex}}=571 \mathrm{~nm}$. The spectra are shown for times, $0,0 \cdot 25,0 \cdot 5,1,4$ and $10 \mathrm{~ns}$. 
Figure 5 shows the TRES and TRANES spectra for nile red in egg-PC membrane. A continuous spectral peak shift by $\sim 10 \mathrm{~nm}$ is observed in the TRES spectra (figure 5a). An isoemissive point is clearly seen in TRANES spectra confirming that the fluorescence emission is due to two emissive species in this case as well.

TRANES analysis of the fluorescence of nile red in the above organized molecular assemblies indicates the presence of two emissive species. However, the identification of

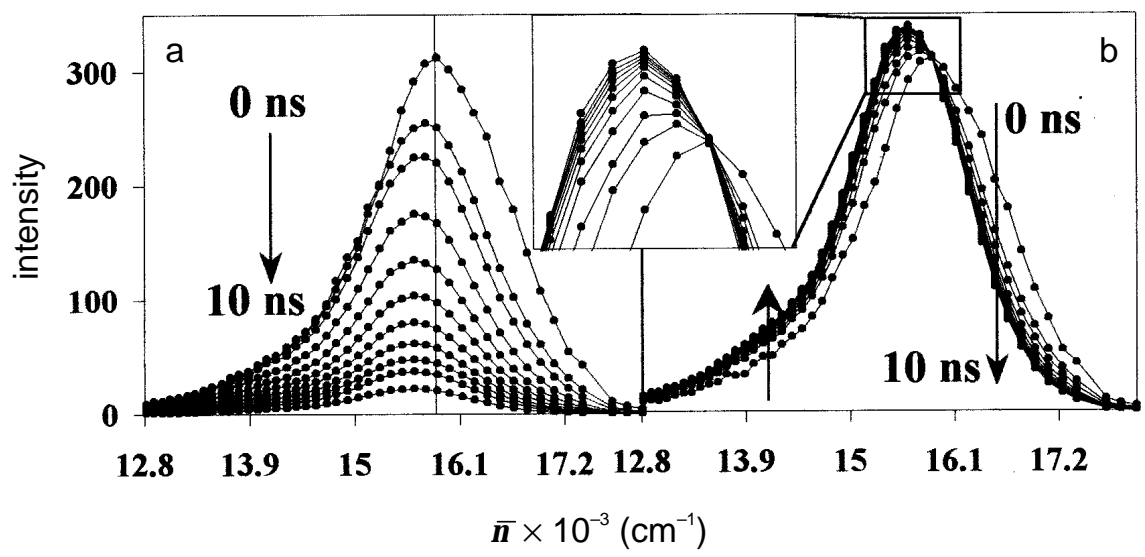

Figure 4. (a) Time-resolved emission spectra (TRES), and (b) time-resolved area normalized emission spectra (TRANES) for nile red in TX-100 micelle. Inset shows the region of isoemissive point in greater detail. Isoemissive point is observed at $630 \mathrm{~nm}$. [dye] $=2 \mu \mathrm{M}$. [TX] $=20 \mathrm{mg} / \mathrm{mL}$. $\lambda_{\mathrm{ex}}=571 \mathrm{~nm}$. The spectra are shown for times, $0,0 \cdot 5,1,2,3,4,5,6,7,8$ and $10 \mathrm{~ns}$.

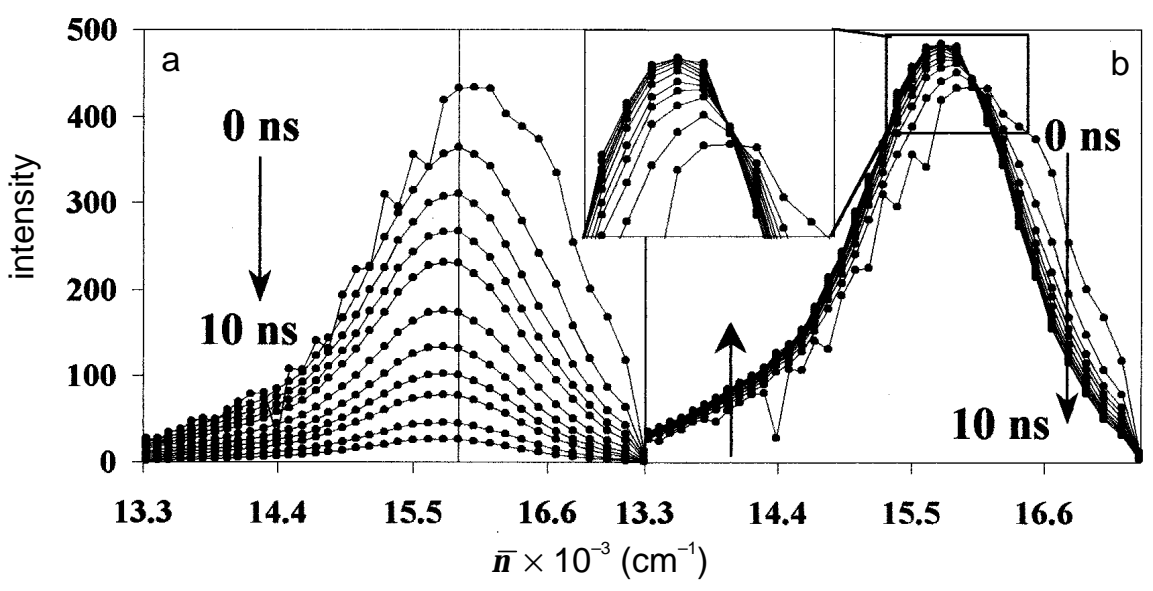

Figure 5. (a) Time-resolved emission spectra (TRES), and (b) time-resolved area normalized emission spectra (TRANES) for nile red in egg-PC membrane. Inset shows the region of isoemissive point in greater detail. Isoemissive point is observed at $625 \mathrm{~nm}$. [dye] $=1 \mu \mathrm{M}$. [egg-PC] $=0 \cdot 2 \mathrm{mg} / \mathrm{mL}$. $\lambda_{\text {ex }}=571 \mathrm{~nm}$. The spectra are shown for times, $0,0 \cdot 5,1,1 \cdot 5,2,3,4,5,6,8$ and $10 \mathrm{~ns}$. 
the nature of the emissive species requires additional experiments and testing specific models that involve two emissive species. Fortunately, there are only two general models that one must consider when there are two emissive species. (I) In the first model, there is only one species in the ground state (say, species A) and the excited state kinetics of $\mathrm{A}^{*}$ produces the second emissive species, $\mathrm{B}^{*}$. In this model, there are no intermediate species between $\mathrm{A}^{*}$ and $\mathrm{B}^{*}$, except the activated complex (or transition state), the concentration of which is negligibly low. The excited state kinetics may be reversible or irreversible. (II) In the second model, there are two spectroscopically distinct species, A and $\mathrm{B}$ in the ground state. Both the species are simultaneously excited giving rise to two emissive species $\mathrm{A}^{*}$ and $\mathrm{B}^{*}$. $\mathrm{A}$ and $\mathrm{B}$, and/or $\mathrm{A}^{*}$ and $\mathrm{B}^{*}$ may be kinetically coupled (e.g., in equilibrium), or they may not be kinetically coupled (e.g., a mixture of two different dyes or the same dye in two different environments in a complex system). For both the models, isoemissive point will be observed in TRANES at the wavelength where the ratio of the radiative rates of the two species is equal to the ratio of their total radiative rates ${ }^{2}$.

Organized molecular assemblies of micelles, model membranes and biological membranes are systems where the physical properties (dielectric constant, fluidity, polarity etc) vary drastically in the length scale of a few Angstroms in the interface region. Hydrophobic organic molecules like nile red are readily incorporated in such assemblies but the location of these molecules in the organized molecular assemblies can be determined only by a detailed set of experiments and analysis ${ }^{4}$. Experiments using fifteen fluorescent dyes incorporated in egg-PC membrane have indicated that three sites of solubilization are adequate for the interpretation of results. These three sites are to be found in, (i) external surface region exposed to water, (ii) interface region, and (iii) hydrophobic core region ${ }^{4}$. It is likely that the dye may not be present in all three sites and prefer only two of the three sites. Considering these points, we conclude that the two emissive species of nile red in micelles and membrane indicated by TRANES analysis may actually be two sites of solubilization for nile red in the above organized molecular assemblies.

Multiexponential fluorescence decays and time-dependent emission spectra in organized molecular assemblies and biological membranes for different probes have been interpreted using models that require the existence of continuous energy states between an initially excited state and the final relaxed one ${ }^{5,6}$. TRANES analysis of time-resolved fluorescence decays would provide unambiguous support for such models. The results of TRANES analysis of several fluorescent dyes in organized molecular assemblies will be published soon.

\section{Conclusions}

TRANES analysis of the fluorescence of nile red in the organized molecular assemblies of micelles and bilayer egg-PC membrane indicates two emissive species in these media. These emissive species are identified with the dye in two different sites of solubilization.

\section{References}

1. Lakowicz J R (ed.) 1992-2000 Topics in fluorescence spectroscopy (New York: Plenum) vol. 1-5 
2. Koti A S R, Krishna M M G and Periasamy N 2001 J. Phys. Chem. 1051767

3. Periasamy N, Doraiswamy S, Maiya B G and Venkataraman B 1988 J. Chem. Phys. 881638

4. Krishna M M G and Periasamy N 1998 J. Fluoresc. 881

5. Hof M 1999 In Applied fluorescence in chemistry, biology and medicine (eds) W Rettig et al (Berlin: Springer) ch. 18

6. Bhattacharyya K and Bagchi B 2000 J. Phys. Chem. 10410603

7. O'Connor D V and Phillips D 1984 Time correlated single photon counting (London: Academic Press) 